\title{
High-gain quantum free-electron laser: Long-time dynamics and requirements
}

\author{
Peter Kling $\odot,{ }^{1,2}$ Enno Giese $\odot,{ }^{2,3}$ C. Moritz Carmesin $\odot,{ }^{2,4}$ Roland Sauerbrey, ${ }^{4}$ and Wolfgang P. Schleich $\oplus^{2,1,5}$ \\ ${ }^{1}$ Institute of Quantum Technologies, German Aerospace Center (DLR), Söflinger Straße 100, D-89077 Ulm, Germany \\ ${ }^{2}$ Institut für Quantenphysik and Center for Integrated Quantum Science and Technology IQ ${ }^{S T}$, Universität Ulm, \\ Albert-Einstein-Allee 11, D-89081, Germany \\ ${ }^{3}$ Institut für Angewandte Physik, Technische Universität Darmstadt, Schlossgartenstr. 7, D-64289 Darmstadt, Germany \\ ${ }^{4}$ Helmholtz-Zentrum Dresden-Rossendorf e.V., Bautzner Landstraße 400, D-01328 Dresden, Germany \\ ${ }^{5}$ Hagler Institute for Advanced Study at Texas A\&M University, Texas A\&M AgriLife Research, Institute for Quantum Science and \\ Engineering (IQSE) and Department of Physics and Astronomy, Texas A\&M University, College Station, Texas 77843-4242, USA
}

(Received 23 March 2021; accepted 27 July 2021; published 10 September 2021)

\begin{abstract}
We solve the long-time dynamics of a high-gain free-electron laser in the quantum regime. In this regime each electron emits at most one photon on average, independently of the initial field. In contrast, the variance of the photon statistics shows a qualitatively different behavior for different initial states of the field. We find that the realization of a seeded quantum free-electron laser is more feasible than self-amplified spontaneous emission.
\end{abstract}

DOI: 10.1103/PhysRevResearch.3.033232

\section{INTRODUCTION}

In the quantum regime of the free-electron laser (FEL) the electrons undergo discrete scattering events instead of following continuous trajectories [1]. More precisely, an electron in the Quantum FEL [2-9] occupies only two discrete momentum levels [10] in analogy to an atomic laser [11].

By identifying three constants of motion we solve in the present paper the long-time dynamics of a Quantum FEL in the high-gain regime, within (i) an analytical approximation and (ii) a numerical simulation. Moreover, we discuss fundamental requirements [12] to realize such a device in an experiment.

Employing momentum-jump operators we showed in Ref. [13] that the dynamics of a high-gain Quantum FEL is effectively governed by the Dicke Hamiltonian [14]. We briefly review this model in Sec. II. In order to solve the resulting equations of motion we applied in Ref. [13] a parametric approximation [15] and obtained an exponential growth of the laser intensity along the wiggler length. However, this approximation breaks down when the number of emitted photons becomes large after a certain interaction time. Therefore, we derive in Sec. III solutions beyond the short-time limit.

As a first result of these studies, we find that in the quantum regime each electron emits at most only a single photon [3], in contrast to the multiphoton processes dominating the classical regime [16]. Moreover, we derive expressions for the saturation length and consider deviations from resonance.

Secondly, we discuss the variance of the photon number. For a startup from vacuum, the field possesses almost chaotic

Published by the American Physical Society under the terms of the Creative Commons Attribution 4.0 International license. Further distribution of this work must maintain attribution to the author $(s)$ and the published article's title, journal citation, and DOI. statistics. In the case of a seeded Quantum FEL, the behavior of the variance depends strongly on the initial field state. A Fock state or a coherent state with a high photon number leads to comparably narrow photon distributions in the course of time. In contrast, the statistics evolving from a thermal state remains broad, but becomes much narrower than a thermal distribution when the intensity assumes its maximal value.

Our results allow us in Sec. IV to identify the challenges for a Quantum FEL experiment, and to explain the necessity for an optical undulator [17,18]. Indeed, it was argued in Ref. [12] that the combined influence of space charge and spontaneous emission into all modes prevents an effective Quantum FEL operation for more than several gain lengths which drastically reduces the feasible laser intensity. We can circumvent this loss of intensity if we consider a seeded FEL instead of selfamplified spontaneous emission (SASE). This effect is a direct consequence of the decreased saturation length in a seeded Quantum FEL so that one drops below the problematic limit put forward in Ref. [12].

Finally, we summarize our main results and conclude in Sec. V. To keep this paper self-contained, we add Appendices $\mathrm{A}$ and $\mathrm{B}$ which are devoted to the detailed calculations associated with our analytical approximation and the numerical approach, respectively.

\section{QUANTUM FEL: BASIC BUILDING BLOCKS}

In Ref. [13] we have formulated the FEL dynamics [19,20] in terms of collective jump operators for the electron momentum. In the following, we review this description, where each electron populates levels on a discrete momentum ladder induced by the quantum mechanical recoil $q \equiv 2 \hbar k$ the electron experiences when it scatters from the fields. Here $\hbar$ denotes the reduced Planck constant while $k$ is the wave number of the laser and wiggler field in the comoving Bambini-Renieri frame [21] where both coincide. 


\section{A. Collective Hamiltonian}

By performing a rotating-wave-like approximation [13] we found that the motion of each electron in the quantum regime is restricted to only two momentum levels, that is, the excited state with momentum $p$, close to the resonant momentum $p=$ $q / 2$, and the ground state with $p-q \cong-q / 2$. In the language of collective operators we therefore defined

$$
\sum_{j=1}^{N}|p-q\rangle^{(j)}\langle p| \equiv \hat{J}_{-} \equiv\left(\hat{J}_{+}\right)^{\dagger}
$$

and

$$
\frac{1}{2} \sum_{j=1}^{N}\left(|p\rangle^{(j)}\langle p|-| p-q\rangle^{(j)}\langle p-q|\right) \equiv \hat{J}_{z},
$$

where we sum over projection operators for all $N$ electrons with $|p\rangle^{(j)}$ denoting the momentum eigenstate of the $j$ th electron.

Indeed, $\hat{J}_{+}, \hat{J}_{-}$, and $\hat{J}_{z}$ satisfy the commutation relations for angular momentum $[22,23]$, that is, $\left[\hat{J}_{+}, \hat{J}_{-}\right]=2 \hat{J}_{z}$ and $\left[\hat{J}_{z}, \hat{J}_{ \pm}\right]= \pm \hat{J}_{ \pm}$. Hence, we identify the jump operators with the ladder operators $\hat{J}_{+}$and $\hat{J}_{-}$of angular momentum, while $\hat{J}_{z}$ describes its $z$ component [24].

The restriction to two momentum levels leads us to the dimensionless Dicke Hamiltonian [14]

$$
\hat{H}_{\text {eff }} \equiv \varepsilon\left(\hat{a}_{\mathrm{L}} \hat{J}_{+}+\hat{a}_{\mathrm{L}}^{\dagger} \hat{J}_{-}\right)-\delta \hat{n},
$$

where the dynamics of the laser mode is described by the photon annihilation, creation, and number operator, $\hat{a}_{\mathrm{L}}, \hat{a}_{\mathrm{L}}^{\dagger}$, and $\hat{n} \equiv \hat{a}_{\mathrm{L}}^{\dagger} \hat{a}_{\mathrm{L}}$, respectively. These field operators fulfill the commutation relation $\left[\hat{a}_{\mathrm{L}}, \hat{a}_{\mathrm{L}}^{\dagger}\right]=1$.

Moreover, we have recalled the dimensionless parameter $\varepsilon \equiv g / \omega_{\mathrm{r}}$ as the ratio of the coupling constant $g$ for electrons and fields [10,25] and the recoil frequency [10] $\omega_{\mathrm{r}} \equiv$ $q^{2} /(2 m \hbar)$ with $m$ denoting the mass of an electron. In addition, $\delta \equiv(p-q / 2) /(q / 2)$ describes the scaled detuning of the initial momentum $p$ of the electrons from resonance at $p=q / 2$. We emphasize that also the time variable $\tau \equiv \omega_{\mathrm{r}} t$ in this description is in a dimensionless form.

The approximation leading to the effective Hamiltonian of Eq. (3) is only valid, provided the quantum parameter $\alpha_{N}$ (compare to Table I) satisfies the inequality

$$
\alpha_{N} \equiv \frac{g \sqrt{N}}{\omega_{\mathrm{r}}} \ll 1,
$$

which defines the quantum regime of the FEL.

Moreover, we require that the detuning from resonance is small, that is, $\delta<1$. For a realistic electron beam, where each electron has a different initial momentum, we additionally require $\Delta p<q$ for the initial momentum spread $\Delta p$ of the electrons.

We emphasize that our single-mode model is indeed very simplified. While this approach seems justified for an FEL seeded by a single mode, it breaks down for the startup from vacuum, where no mode of the radiation field is preferred and we know from classical SASE that a comparably broad spectrum is emitted [26-28]. To gain some insights into the subtleties of SASE in the quantum regime, we nevertheless use this model as a starting point in rough analogy to the development of classical FEL theory, where corrections due to various effects were introduced step by step [29].

\section{B. Constants of motion}

Despite several attempts [22,30-35], no closed analytic solution for the dynamics dictated by the Dicke Hamiltonian has been found. Hence, we resort to approximations and numerical methods. Hereby, we closely follow the lines of the existing literature $[22,32,33,36]$. In addition, we study the effect of a nonzero detuning from resonance.

The key ingredient of our approach is the identification of three constants of motion [32]. From the Hamiltonian in Eq. (3) we straightforwardly derive that the total angular momentum

$$
\hat{A} \equiv \hat{J}^{2}=\frac{1}{2}\left(\hat{J}_{+} \hat{J}_{-}+\hat{J}_{-} \hat{J}_{+}\right)+\hat{J}_{z}^{2}
$$

as well as the total number of excitations

$$
\hat{B} \equiv \hat{n}+\hat{J}_{z}
$$

are constants of motion which commute with each other, that is, $[\hat{A}, \hat{B}]=0$.

Moreover, the Hamiltonian

$$
\hat{C} \equiv \hat{H}_{\mathrm{eff}}
$$

is independent of time and thus itself constitutes a third constant.

\section{Our approaches}

We use these quantities to investigate the long-time dynamics of a high-gain Quantum FEL in an analytical as well as a numerical approach. The former one is carried out in detail in Appendix A and relies [32] on the decoupling of the Heisenberg equations of motion of the photon-number operator $\hat{n}$ with the help of $\hat{A}, \hat{B}$, and $\hat{C}$. To solve the resulting differential equation of noncommuting operators, we approximate the operators by $c$-numbers $[32,33]$. By this procedure we find an analytical expression for the mean photon number $n \equiv\langle\hat{n}\rangle$ in terms of a Jacobi elliptic function [22,33,37].

In contrast, the approach of Appendix B towards a numerical solution [36] is based on the evolution of time-dependent state vectors as solutions of the Schrödinger equation. For that, we expand the state of the laser field into Fock states $|n\rangle$ with photon number $n$ while the collective state of the electrons is described by the basis $|r, m\rangle$, where $r$ corresponds to the total angular momentum and $m$ to its $z$ component. Since $\hat{A}$ and $\hat{B}$ are constant, the set $n, r, m$ of three independent quantum numbers is reduced to a single one. As a consequence, the Schrödinger equation leads to a three-term recurrence relation for the expansion coefficients which we straightforwardly solve by the numerical diagonalization of a tridiagonal matrix of dimension $(N+1) \times(N+1)$.

\section{LONG-TIME DYNAMICS}

In the following we present our results of the analytical approximation and the numerical simulation for the long-time dynamics of a high-gain Quantum FEL. Here we first focus on the time evolution of the mean number of photons and 
concentrate on the dependence of the saturation intensity and length on the number of electrons. We conclude by discussing the variance of the photon distribution of the Quantum FEL.

\section{A. Time evolution of mean photon number}

In Appendix A we derive the approximate expression

$$
n(L)=n_{0}+\left(n_{+}-n_{0}\right) \mathrm{cn}^{2}\left[\frac{L}{2 L_{g}} \sqrt{\frac{n_{+}+n_{-}}{N}}-K, \mathfrak{K}\right]
$$

for the mean photon number $n \equiv\langle\hat{n}\rangle$ of a Quantum FEL as a function of the length $L$ of the wiggler, where cn denotes a Jacobi elliptic function [37]. The quantities $n_{+}$and $n_{-}$are roots of the right-hand side of the differential equation (A9) for $n$. The explicit expressions for these roots are given in Eq. (A10) and depend on the parameters of this differential equation, that is, the initial photon number $n_{0}$, the electron number $N$, and the detuning $\delta / \alpha_{N}$ from resonance in units of the quantum parameter.

Moreover, we have recalled from Refs. [3,13] the gain length

$$
L_{g} \equiv \frac{c}{2 g \sqrt{N}}
$$

of a Quantum FEL.

In addition, the Jacobi elliptic function cn is characterized by the modulus $\mathfrak{K}=\mathfrak{K}\left(n_{+}, n_{-}\right)$from Eq. (A15), whereas $K \equiv$ $K(\mathfrak{K})$ describes a complete elliptic integral of first kind [37].

We emphasize that the initial state of the laser field enters in the approximation, Eq. (8), only through the mean photon number $n_{0}$. In Fig. 1 we compare the analytical approximation for the mean photon number $n=\langle\hat{n}\rangle$ to the numerical solution, for exact resonance $p=q / 2$ and with $N=10^{4}$ electrons [38]. Here we consider the startup from vacuum (top) despite the limitations of our single-mode model as well as a seeded FEL evolving from a Fock state with $n_{0}=10^{3}$ photons (bottom).

In both cases we observe an exponential startup in accordance with Ref. [13]. However, this growth saturates for increasing values of the wiggler length $L$ leading to a local maximum of the photon number. The mean photon number then decreases until it reaches its initial value, before it again increases in an oscillatory-like behavior.

For startup from vacuum we deduce from the analytical approach that each electron emits at most one photon, that is, $n_{\max }=N$, in contrast to the smaller value $n_{\max } \cong 0.8 N$ obtained by the numerical simulation. The second maximum of the numerical solution, however, is even more suppressed compared to the analytical approximation.

We interpret these deviations as the result of entangled Dicke states for the electron momenta $[13,14]$ which the numerical solution takes into account. The oscillations of the analytical solution between 0 and $N$ indicate that in this model all electrons are in the ground state when the maximum photon number is reached. In the exact treatment, however, the electrons become entangled due to their common interaction with the laser field. This entanglement prevents a product state, where each electron is in the ground state, decreasing the maximum photon number.

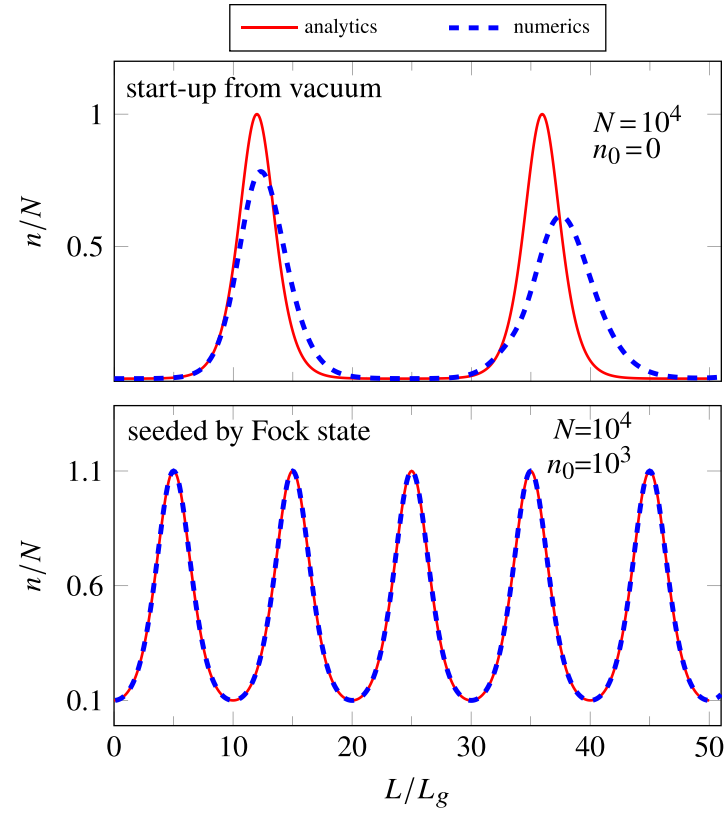

FIG. 1. Mean photon number $n=\langle\hat{n}\rangle$ of a high-gain Quantum FEL as a function of the wiggler length $L$ scaled in units of the gain length $L_{g}$. We compare the analytical approximation (red line) from Eq. (8) to the numerical solution (blue, dashed line) of Eq. (B4) for the startup from vacuum (top), that is, $n_{0}=0$, as well as for a seeded FEL (bottom) with $n_{0}=10^{3}$ photons in an initial Fock state. For both plots we have assumed resonance, $p=q / 2$, and the value $N=10^{4}$ for the number of electrons. In all cases the curves show an exponential growth for short times which saturates and leads to a first maximum followed by further oscillations. For the startup from vacuum the photon number saturates at about ten gain lengths. There, the approximate solution takes on the value $n=N$, that is, each electron has emitted exactly one photon, while the numerically computed maximum is at about $n \cong 0.8 N$. In addition, the locations of these maxima for analytics and numerics are slightly shifted with respect to each other. For an FEL seeded by a large Fock state, numerics and analytics agree very well. In this case, we obtain complete oscillations of the mean photon number between the values $n_{0}$ and $n_{0}+N$ with a significantly shorter periodicity than for the startup from vacuum.

Despite these differences between the numerical and the approximate result, the latter one is helpful to estimate the qualitative behavior of the dynamics. This feature becomes even more important in the discussion of experimentally relevant length scales in Sec. IV, when we increase the electron number $N$ to more realistic values where a numerical diagonalization becomes impracticable. In this limit, we have to resort to the predictions given by the expression in Eq. (8).

In contrast, for a seeded Quantum FEL the numerical and analytical solution for the mean photon number agree very well, at least for a Fock state. In this situation we observe oscillations of the mean photon number between $n_{0}$ and $n_{0}+N$. We note that the periodicity of these oscillations is much shorter than for the startup from vacuum.

So far, we have only considered the case of the initial state of the laser field being given by a Fock state. We now study the influence of the photon statistics of the initial state on 


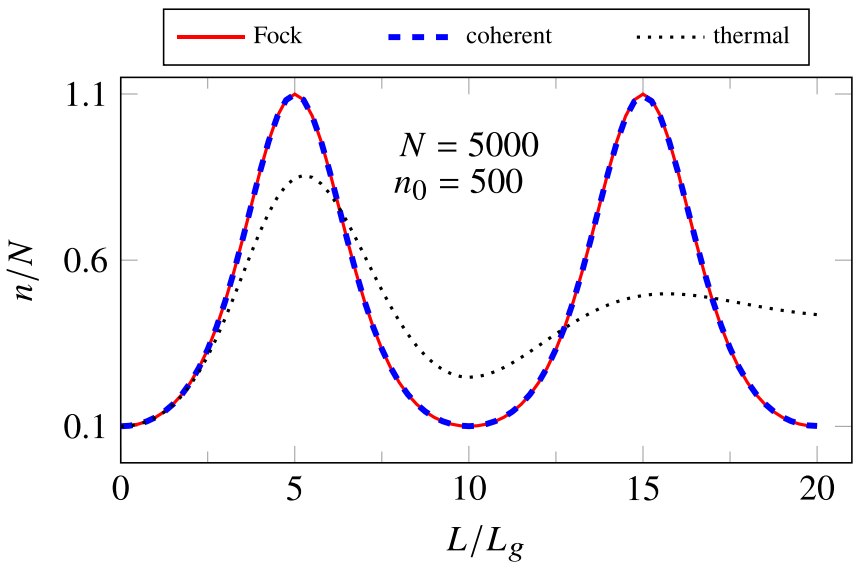

FIG. 2. Mean photon number $n=\langle\hat{n}\rangle$ of a seeded high-gain Quantum FEL as a function of the wiggler length $L$ in units of the gain length $L_{g}$. We depict the numerical solution for $n$ with respect to three different initial states for the laser field, that is, a Fock state (red line), a coherent state (blue, dashed line), and a thermal state (black, dotted line). The latter two states are described by the photon distributions in Eqs. (B6) and (B7). In all three examples we have chosen the values $n_{0}=500$ and $N=5000$ for the initial mean photon number and the number of electrons, respectively. While a Fock state and a coherent state lead to the same oscillatory behavior of the photon number between $n_{0}$ and $N+n_{0}$, the situation for a thermal state is different. Here the first maximum of $n$ attains the decreased value of approximately $0.8 N$ instead of $1.1 N$. Moreover, we do not observe full oscillations, but the photon number seems to approach a constant value of approximately $0.5 \mathrm{~N}$.

the time evolution of the mean photon number and refer to Appendix B for details.

Therefore, we show in Fig. 2 the numerically evaluated expectation value $n=\langle\hat{n}\rangle$ as a function of $L$, with the initial state of the field given by (i) a Fock state, (ii) a coherent state, and (iii) a thermal state. To make a meaningful comparison we have chosen in the three cases the same initial mean photon number $n_{0}=500$ while assuming $N=5000$ electrons. A coherent state is usually employed to model the output of a coherent light source such as a laser. In contrast, a thermal state describes a random, incoherent source, for example, a light bulb [39,40].

We observe that a coherent state leads approximately to the same behavior as a Fock state, that is, an oscillation of the photon number between $n_{0}$ and $N+n_{0}$. However, the curve corresponding to a thermal input state is different. Here the maximum photon number reaches only the decreased value of $0.8 \mathrm{~N}$ instead of $1.1 \mathrm{~N}$. In addition, the oscillatory behavior is washed out and $n$ seems to approach a constant value of about $0.5 N$.

Similar to a Fock state, a coherent state with a high photon number possesses a sharply peaked photon distribution $p_{n}$. We obtain the mean photon number through

$$
n(L)=\sum_{n^{\prime}=0}^{\infty} p_{n^{\prime}} n_{n^{\prime}}(L)
$$

expressed by Eq. (B15). Here $n_{n^{\prime}}=n_{n^{\prime}}(L)$ denotes the mean photon number for the initial Fock state $n^{\prime}$.
Hence, mainly contributions with photon numbers close to the mean $n_{0}$ are relevant. Since the dynamics of these contributions occurs on similar timescales, the averaging process yields results close to those originating from a delta-peaked photon distribution, that is, for a Fock state.

In contrast, the photon distribution of a thermal state is very broad and, moreover, is not symmetric around $n_{0}$ [40]. Hence, we have to average over many differently weighted contributions which corresponds to many different timescales of the resulting dynamics.

Additionally, the probabilities $p_{n}$ for a thermal state increase for photon numbers close to zero. There, the dynamics of $n$ is drastically different from high initial photon numbers as apparent in Fig. 1. This mixing of many different timescales, combined with the influence of the qualitatively different behavior for small photon numbers, explains the difference in Fig. 2 between a thermal input state and a coherent or a Fock state.

\section{B. Saturation intensity and length}

In this section we investigate the magnitude $n_{\max }$ and the position $L_{\max }$ of the first maximum of the mean photon number shown in Fig. 1, that is, the saturation intensity and the saturation length, respectively, of a Quantum FEL. Our analysis distinguishes the two cases of exact resonance and near resonance.

\section{Resonant case}

For the time being, we consider exact resonance, that is, $p=q / 2$, and assume that the laser field is initially described by a Fock state. From Eqs. (8) and (A10) we derive the simple relation $n_{\text {max }} \cong n_{0}+N$ for the maximum photon number which we have already deduced from Fig. 1. There, we have also found that for $n_{0}=0$ the numerical result is lower than the estimated one, at least for our choice of $N=10^{4}$ electrons.

In the upper part of Fig. 3 we now examine if this discrepancy between analytics and numerics continues to exist for different values of $N$ by plotting the numerically evaluated result for $n_{\max }$ against $N$. In the limit of a single electron exactly one photon is emitted in accordance with the single-electron approach of Ref. [10]. Here Rabi oscillations between excited and ground state occur and no entangled Dicke states, which we have identified as the reason for a decreased ratio $n_{\max } / N$, emerge.

However, already for low electron numbers the value $n_{\text {max }} \cong 0.78 N$ is reached which then remains constant for the whole range of the considered values for $N$. We therefore deduce that (i) this behavior continues for increasing values of $N$ where numerics starts to become impracticable, and that (ii) the analytical result $n_{\max }=N$ provides us at least with the correct order of magnitude. Already in Ref. [31] it was predicted that the maximum photon number $n_{\max }$ resulting from the Dicke Hamiltonian with $N \gg 1$ atoms varies between $n_{\text {max }} \cong n_{0}+N$ for a large initial photon number $n_{0} \gg 1$ and $n_{\text {max }} \cong 4 / 5$ for $n_{0}=0$ in accordance with our numerical solution. Note that these results have been obtained within our singe-mode model, whose predictive power is limited for a startup from vacuum.

In Fig. 1 we have also observed a different $L_{\max }$ for the startup from vacuum compared to the seeded case. To find 


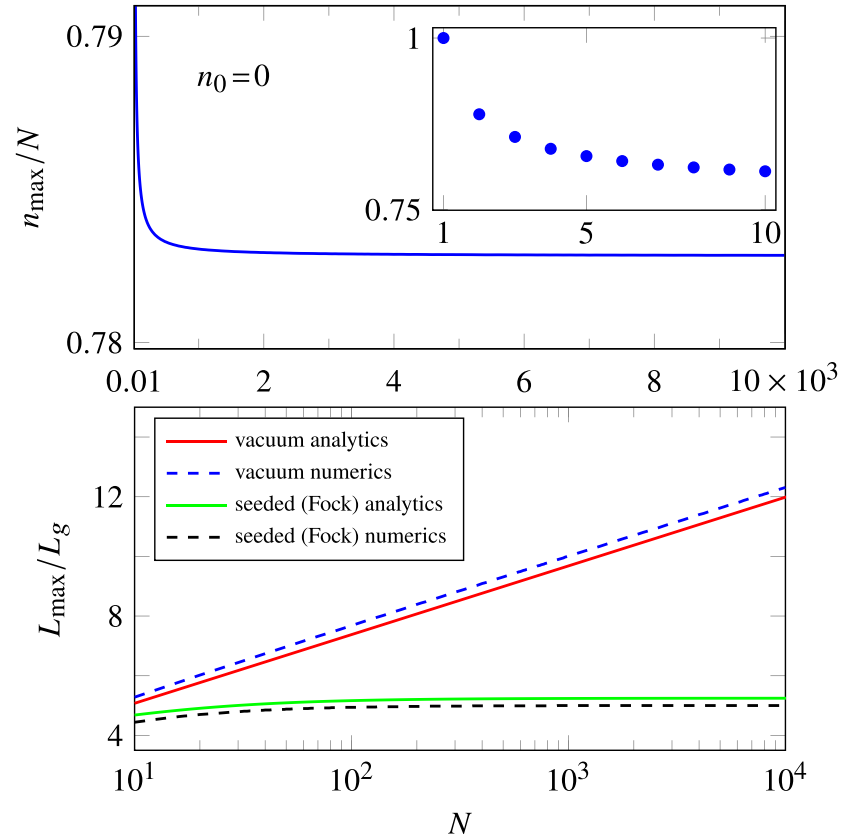

FIG. 3. The first maximum $n_{\max }$ (top) of the photon number and the corresponding saturation length $L_{\max }$ (bottom), both as functions of the number $N$ of electrons and for resonance $p=q / 2$. In the top panel, which covers only the startup from vacuum with $n_{0}=$ 0 , we observe that $n_{\max }$ very quickly reaches the constant value of $n_{\max }=0.78 \mathrm{~N}$ in contrast to the analytical approximation which predicts $n_{\max }=N$. The inset shows the behavior of $n_{\max }$ for very small values of $N$. For $N=1$ we find that the electron emits one photon due to the Rabi oscillation in the single-electron approach of Ref. [10]. At the bottom, the comparison of analytics, Eq. (11) (red line) to numerics (blue, dashed line) reveals the same logarithmic behavior of $L_{\max }$ apart from a small shift between the two curves, if we consider startup from vacuum, $n_{0}=0$. In the case of a Quantum FEL seeded with a Fock state and with a fixed ratio of $n_{0} / N=0.1$ analytics (green line) and numerics (black, dashed line) also agree well. Here both curves predict the constant value $L_{\max } \cong 5 L_{g}$ with $L_{g}$ denoting the gain length from Eq. (9).

an analytical expression for $L_{\max }$ we now take a closer look at the elliptic function $\mathrm{cn}$ in Eq. (8). The first maximum of this function occurs, when its arguments vanish. With the help of this condition and the asymptotic behavior [37] of $K$ in Eq. (A13) for $\mathfrak{K}=1-\mathcal{O}\left(N^{-1}\right) \rightarrow 1$ we arrive at the expression

$$
\frac{L_{\max }}{L_{g}} \cong 2 \ln \left(4 \sqrt{\frac{N}{n_{0}+1}}\right)
$$

for the saturation length with $p=q / 2$ and $N \gg 1$ [41].

For $n_{0}=0$ we thus read off a logarithmic growth of $L_{\max }$ with the electron number $N$, that is, $L_{\max } \propto \ln N$. In the case of a seeded Quantum FEL with $n_{0} \gg 1$ the important parameter that determines the magnitude of $L_{\max }$ is the ratio of electron number $N$ and initial photon number $n_{0}$. This scaling explains the difference in Fig. 1 of $L_{\max }$ for a seeded FEL, and one starting from vacuum because of $N / n_{0} \ll N$.

In the lower part of Fig. 3 we depict $L_{\max }$ as a function of $N$. Here we compare the analytical expression in Eq. (11)

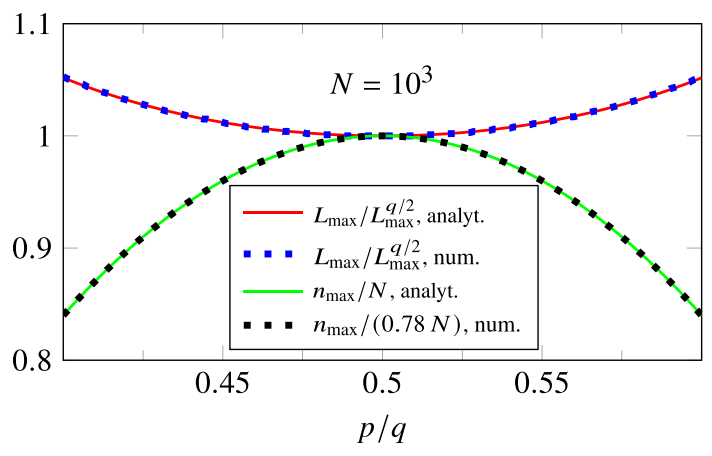

FIG. 4. Saturation length $L_{\max }$ (upper curves) and the corresponding maximum photon number $n_{\max }$ (lower curves), as functions of the momenta $p$ in the neighborhood of resonance for $N=10^{3}$ electrons and for $n_{0}=0$. We observe that $n_{\max }$ decreases and $L_{\max }$ increases for a growing deviation from resonance $p=q / 2$. For both, $L_{\max }$ and $n_{\max }$, we find perfect agreement between analytics, that is, the red curve and the green curve, respectively, and numerics, that is, the blue dotted and the black dotted curve, respectively. We emphasize that the analytical and the numerical curves are normalized to their respective values at resonance which, however, differ from each other. For example, the analytical result for $n_{\max }$ is divided by $N$ while its numerical counterpart is divided by $0.78 N$.

to the numerical simulation. For the startup from vacuum we observe that both curves show the same behavior, apart from a small shift, already apparent in Fig. 1. Hence, we infer that the analytical approximation gives a reasonable estimate also for $L_{\max }$. For a seeded FEL with the fixed ratio $n_{0} / N=0.1$, the numerical and the analytical curve also match very well with both predicting a constant value of $L_{\max }$, in accordance with Eq. (11).

\section{Off-resonant case}

We now study the dependence of the maximum photon number on the detuning of the momentum of the electrons from resonance. For that, we restrict ourselves to the startup from vacuum $n_{0}=0$.

According to the analytical solution from Eq. (8), the maximum photon number $n_{\max }$ is given by the elementary relation

$$
n_{\max }=n_{+} \cong N\left(1-\frac{\delta^{2}}{4 \alpha_{N}^{2}}\right)
$$

valid for $N \gg 1$, where we have used the expression for $n_{+}$ from Eq. (A10).

Thus, $n_{\max }$ is different from zero only for $-2 \alpha_{N}<\delta<$ $2 \alpha_{N}$, which gives rise to a gain bandwidth of $q /\left(2 \alpha_{N}\right)$ in momentum space, in accordance with the exponential-gain regime [13]

Moreover, from Eq. (A13) we derive for $N \gg 1$ the asymptotic expression

$$
\frac{L_{\max }}{L_{g}}=\frac{1}{\sqrt{1-\frac{\delta^{2}}{4 \alpha_{N}^{2}}}}\left[\ln N+4 \ln 2+2 \ln \left(1-\frac{\delta^{2}}{4 \alpha_{N}^{2}}\right)\right]
$$

for the saturation length.

In Fig. 4 we show both $n_{\max }$ and $L_{\max }$ as a function of $p$ which reveals a perfect agreement between our analytical results and numerics. We note that $n_{\max }$ decreases for an 


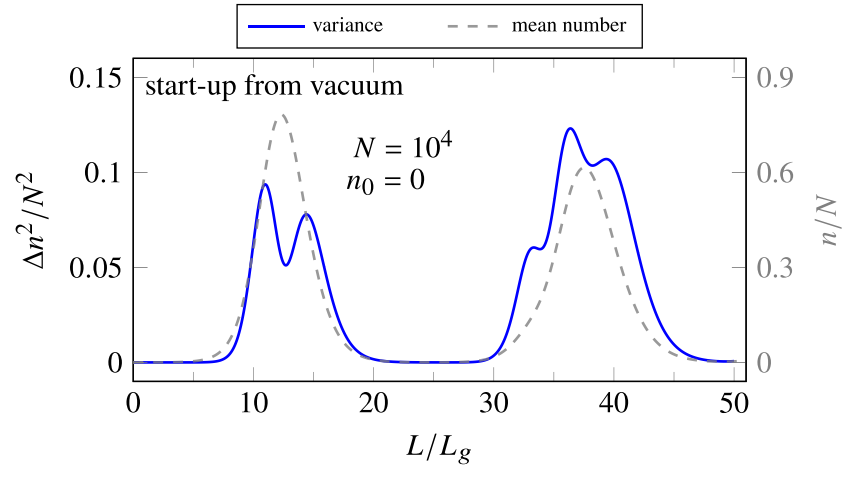

FIG. 5. Variance $\Delta n^{2}$ of the photon-number distribution in a high-gain Quantum FEL as a function of the wiggler length $L$ scaled in units of the gain length $L_{g}$. For the underlying numerical simulation we have assumed resonance $p=q / 2, N=10^{4}$ electrons, and startup from vacuum, that is, $n_{0}=0$. We obtain a qualitatively similar behavior as for the mean photon number (compare to gray, dashed line and right axis), that is, exponential growth, local maximum, and decrease in an oscillatory-like fashion. However, the structure of $\Delta n^{2}$ is more complicated. For example, close to $L_{\max }$ a dip occurs, where $\Delta n^{2} \cong 0.05 N^{2}$ while $n_{\max } \cong 0.8 N$. Hence, the value of $\Delta n^{2}$ is smaller, but roughly of the order of magnitude of $n_{\max }^{2}$ corresponding to an almost chaotic behavior of the laser field.

increasing deviation from resonance $p=q / 2$, while the value of $L_{\max }$ increases.

\section{Variance of photon number}

A quantum mechanical observable is not only characterized by its mean value, but also by its higher moments. The numerical solution of Eq. (B4) enables us to calculate these moments for the observables of a high-gain Quantum FEL. We now study the second moment, that is, the variance of the photon number which is a measure of the intensity fluctuations of the emitted radiation.

In Fig. 5 we depict the variance

$$
\Delta n^{2} \equiv\left\langle\hat{n}^{2}\right\rangle-\langle\hat{n}\rangle^{2}
$$

of the photon number as a function of the wiggler length $L$ for resonance $p=q / 2$, and for the startup from vacuum with $N=10^{4}$ electrons. Similar to the mean value in Fig. 1, the variance shows an oscillatory behavior [31]. However, compared to the mean value (dotted line) the curve corresponding to the variance displays a richer structure with a dip close to $L_{\text {max }}$. Here we find $\Delta n^{2} \cong 0.05 N^{2}$ while $n_{\max } \cong 0.8 N$. Hence, the variance is smaller, but of the same order of magnitude, compared to the square of the mean value and we deduce a nearly chaotic behavior of the radiation field [15].

The situation, however, changes, when we consider a seeded FEL illustrated in Fig. 6. Here we compare the Fano factor [39] $\sigma^{2} \equiv \Delta n^{2} /\langle\hat{n}\rangle$ depending on the wiggler length $L$ for two different initial states of the radiation field, that is, a Fock state [31] and a coherent state characterized by the same mean number of photons.

While the variance for a Fock state vanishes, a coherent state has Poissonian photon statistics with $\Delta n^{2}=\langle\hat{n}\rangle$. As time evolves we obtain in both cases super- but also sub-Poissonian

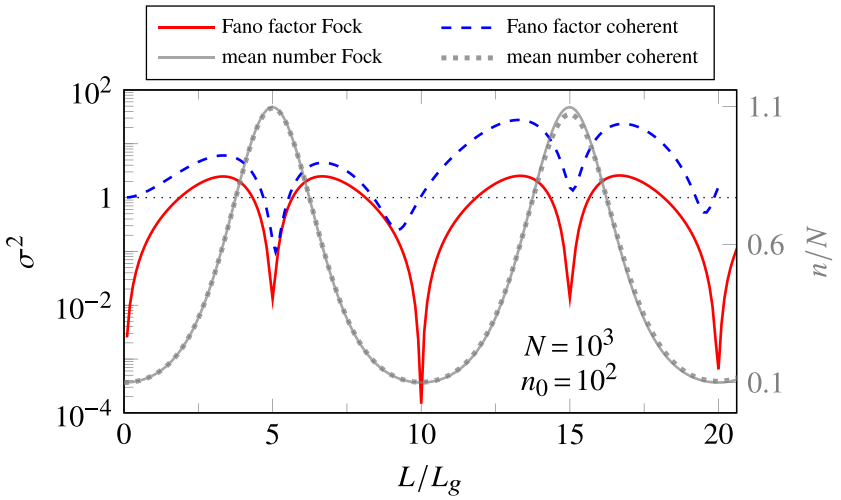

FIG. 6. Fano factor $\sigma^{2} \equiv \Delta n^{2} /\langle\hat{n}\rangle$ of the photon-number distribution in a seeded high-gain Quantum FEL as a function of the wiggler length $L$ scaled in units of the gain length $L_{g}$ for two different initial states, that is, a Fock state (red line) and a coherent state (blue, dashed line). For the underlying numerical simulations we have assumed resonance $p=q / 2, N=10^{3}$ electrons, and an initial mean photon number of $n_{0}=10^{2}$. In both cases we observe super- as well as sub-Poissonian behavior which are separated by the horizontal dotted line at $\sigma^{2}=1$. We note that the first minimum of this normalized variance occurs in the vicinity of the first maximum of the mean photon number (compare to the gray solid and dotted lines as well as to the right axis) at $L \cong 5 L_{g}$. Moreover, we find that the fluctuations for the initially coherent case drastically increase at around ten gain lengths, while only slowly increasing for the case of a Fock state.

photon statistics, in contrast to the broad distribution originating from the startup from vacuum displayed in Fig. 5. Both curves show sub-Poissonian statistics close to the first maximum of the mean photon number indicating a nonclassical state of light. Moreover, we obtain a drastic increase of the fluctuations for larger values of $L$, when the field initially was in a coherent state. In contrast, for an initial Fock state a growth of the fluctuations is hardly visible.

In Fig. 7 we study the second moment of the field when it starts from a thermal state where $\Delta n^{2}=\langle\hat{n}\rangle^{2}$. Although the variance of the photon number remains of the same order of magnitude as the square of the mean value, the corresponding ratio attains a prominent minimum close to the saturation length where the mean intensity assumes a maximum. Here the corresponding statistics with $\Delta n^{2}<\langle\hat{n}\rangle^{2}$ deviates significantly from the thermal distribution of the initial state. Hence, the intensity noise of the emitted light is at least partially decreased.

\section{EXPERIMENTAL REQUIREMENTS}

In this section we address the challenges associated with realizing a Quantum FEL. Already from our elementary onedimensional description we can establish the most relevant experimental conditions. Constraints which go beyond the limits of our model are only mentioned here, but are studied in more detail in Ref. [12].

In Table I we have summarized the important parameters of our model of a Quantum FEL in the Bambini-Renieri frame as well as in the laboratory frame [42]. In addition, we have expressed these quantities in terms of the "universal scaling" 


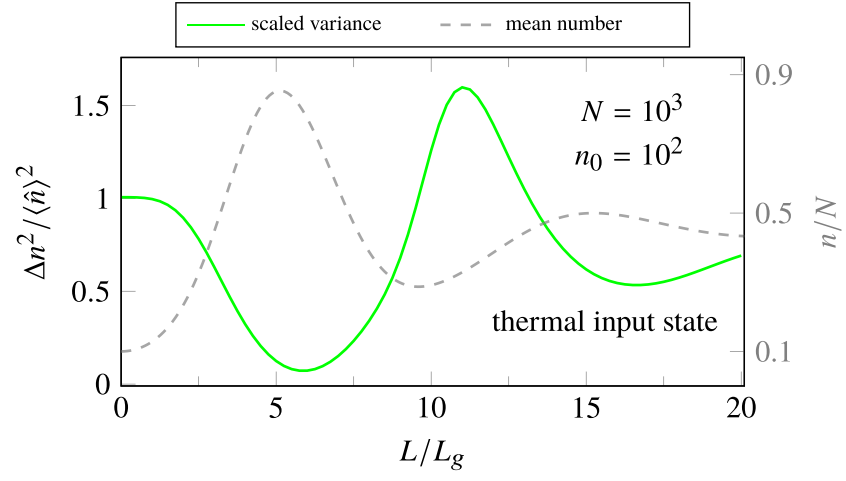

FIG. 7. Variance $\Delta n^{2}$ of the photon number in a seeded high-gain Quantum FEL as a function of the wiggler length $L$ scaled in units of the gain length $L_{g}$. We have normalized the variance with respect to $\langle\hat{n}\rangle^{2}$ to investigate the evolution of the fluctuations in an initial thermal state. The variance $\Delta n^{2}$ is of the same order of magnitude as $\langle\hat{n}\rangle^{2}$. However, this ratio attains a significant minimum when the first maximum of the mean photon number is reached (compare to gray, dashed line and right axis).

of Ref. [3], where $\bar{\rho} \equiv \rho m c / q$ with the Pierce parameter $\rho$ [26], is the analog of our quantum parameter $\alpha_{N}$ [43].

\section{A. Need for optical undulator}

From Table I we deduce the condition

$$
1 \gg \alpha_{N} \propto a_{0}\left(1+a_{0}^{2}\right)^{3 / 2} \frac{\lambda_{\mathrm{W}}^{5 / 2} n_{\mathrm{e}}^{1 / 2}}{\gamma_{0}^{3}}
$$

for the quantum regime in the laboratory frame, where $n_{\mathrm{e}}$ denotes the electron density while $a_{0}, \lambda_{\mathrm{W}}$, and $\gamma_{0}$ are the wiggler parameter, the wavelength of the wiggler in the laboratory frame, and the ratio of the kinetic energy to the rest energy of an electron, respectively.
This relation implies that either increasing the electron energy $\gamma_{0}$, or lowering the wiggler wavelength $\lambda_{\mathrm{W}}$ leads to a decreasing value of the quantum parameter.

At the same time, we have to ensure that the gain length

$$
L_{g} \propto \frac{1}{a_{0}\left(1+a_{0}^{2}\right)^{1 / 2}} \frac{\gamma_{0}^{2}}{\lambda_{\mathrm{W}}^{1 / 2} n_{\mathrm{e}}^{1 / 2}}
$$

given in Table I does not become unfeasibly large.

We observe that high $\gamma_{0}$ as well as small $\lambda_{\mathrm{W}}$ lead to a large gain length. However, while the scaling of $L_{g}$ with the energy $\gamma_{0}$ is quadratic, the dependence on the undulator wavelength scales only with $\lambda_{\mathrm{W}}^{-1 / 2}$. Hence, in order to satisfy Eq. (15) and at the same time minimizing the gain length $L_{g}$, Eq. (16), we propose to operate a Quantum FEL with a small undulator wavelength and with a moderately high electron energy [44] resulting in the emission of $\mathrm{x}$-ray radiation due to $\lambda_{\mathrm{L}} \sim \lambda_{\mathrm{W}} / \gamma^{2}[26]$.

The requirement of a small undulator wavelength quite naturally forces us to employ an optical undulator [17], where the periodic array of magnets is replaced by counterpropagating laser fields $[17,18,45,46]$. For an efficient interaction between electrons and wiggler we also need according to Eq. (16) a relatively large value of the wiggler parameter $a_{0}$, that is, a high intensity of the counterpropagating "pump" laser.

However, a laser with the required intensity would not operate in a continuous way, but rather in a pulsed mode which decreases the interaction length. To overcome this problem the authors in Ref. [18] proposed a "traveling-wave Thompson scattering" (TWTS) scheme. Instead of the usual head-on geometry for electrons and optical undulator, TWTS uses a side-scattering geometry, where a laser pulse with a tilted front interacts under an optimal angle with the electron beam. This procedure can considerably enhance the interaction length.

TABLE I. Parameters of a Quantum FEL in the Bambini-Renieri (BR) frame (left), in the universal scaling of Ref. [3] (center), and in the laboratory frame (right). We consider the five adjustable parameters wavelength $\lambda_{\mathrm{W}}$ of the undulator, its dimensionless field amplitude $a_{0}$, electron density $n_{\mathrm{e}}$, dimensionless energy $\gamma_{0}$ of the electrons, and associated spread $\Delta \gamma_{0}$. Here, $\lambda_{\mathrm{C}}$ and $r_{\mathrm{e}}$ are the Compton wavelength of the electron and the classical electron radius, respectively. In addition, we have introduced the longitudinal dimensionless energy $\gamma$ of the electrons

\begin{tabular}{|c|c|c|c|c|}
\hline & & BR frame & Universal scaling & Laboratory frame \\
\hline Quantum parameter & $\alpha_{N}$ & $\frac{g \sqrt{N}}{\omega_{\mathrm{r}}}$ & $\bar{\rho}^{3 / 2}$ & $\frac{\sqrt{r_{\mathrm{e}} n_{\mathrm{e}}}}{32 \gamma_{0}^{3} \sqrt{\pi}} \frac{\lambda_{\mathrm{W}}^{5 / 2}}{\lambda_{\mathrm{C}}^{3 / 2}} a_{0}\left(1+a_{0}^{2}\right)^{3 / 2}$ \\
\hline Momentum spread & & $\Delta p<2 \hbar k$ & $\frac{\Delta \gamma}{\gamma}<\frac{\rho}{\bar{\rho}}$ & $\frac{\Delta \gamma_{0}}{\gamma_{0}}<\frac{4 \gamma_{0}}{1+a_{0}^{2}} \frac{\lambda_{\mathrm{C}}}{\lambda_{\mathrm{W}}}$ \\
\hline Quantum gain length & $L_{g}$ & $\frac{c}{2 g \sqrt{N}}$ & $\frac{1}{\sqrt{\bar{\rho}}} \frac{\lambda_{\mathrm{W}}}{4 \pi \rho}$ & $\frac{\gamma_{0}^{2}}{\sqrt{\pi} a_{0} \sqrt{1+a_{0}^{2}}} \sqrt{\frac{\lambda_{\mathrm{C}} / \lambda_{\mathrm{W}}}{r_{\mathrm{e}} n_{\mathrm{e}}}}$ \\
\hline Classical gain length & $L_{g}^{\mathrm{cl}}$ & $\frac{c}{\sqrt{3}}\left[\frac{2}{g^{2} \omega_{\mathrm{r}} N}\right]^{1 / 3}$ & $\frac{1}{\sqrt{3}} \frac{\lambda_{\mathrm{W}}}{4 \pi \rho}$ & $\frac{1}{\sqrt{3}} \frac{\gamma_{0} \lambda_{\mathrm{W}}^{1 / 3}}{2 \pi^{2 / 3}\left(a_{0}^{2} r_{\mathrm{e}} n_{\mathrm{e}}\right)^{1 / 3}}$ \\
\hline Plasma wave number & $k_{\mathrm{p}}$ & $\sqrt{\frac{e^{2} n_{\mathrm{e}}}{\varepsilon_{0} m c^{2}}}$ & $\frac{4 k_{\mathrm{W}}}{a_{0}} \rho^{3 / 2}$ & $\sqrt{\frac{4 \pi r_{\mathrm{e}} n_{\mathrm{e}}}{\gamma_{0}^{3}}}$ \\
\hline Spontaneous decay rate & $R_{\mathrm{sp}}$ & $\frac{2 \pi}{3} \frac{r_{\mathrm{e}}}{\lambda_{\mathrm{C}}} a_{0}^{2} k$ & & $\frac{(2 \pi)^{2}}{3} \frac{a_{0}^{2} r_{\mathrm{e}}}{\lambda_{\mathrm{W}} \lambda_{\mathrm{C}}}$ \\
\hline
\end{tabular}
in the wiggler which is connected by the relation $\gamma=\gamma_{0}\left(1+a_{0}^{2}\right)^{-1 / 2}$ to the free energy and the wiggler parameter. 


\section{B. Electron beam}

Apart from the condition in Eq. (15) on the quantum parameter $\alpha_{N}$ which has led to constraints for the wiggler, we have the inequality $\Delta p<q$ for the momentum spread of the electrons which reflects itself in the required quality of the electron beam. According to Table I this condition translates into the inequality

$$
\frac{\Delta \gamma_{0}}{\gamma_{0}}<\frac{4 \gamma_{0}}{1+a_{0}^{2}} \frac{\lambda_{\mathrm{C}}}{\lambda_{\mathrm{W}}}
$$

for the relative energy spread of the electron beam in the laboratory frame with $\lambda_{\mathrm{C}}$ denoting the Compton wavelength of the electron.

Nevertheless, we note that also here a short wiggler wavelength is advantageous since it raises the upper bound of the inequality. Equation (17) represents an ambitious requirement on the quality of the electron beam $[10,47]$.

Indeed, for an efficient operation of a Quantum FEL the momentum spread $\Delta p$ has to be smaller than the gain bandwidth $2 \alpha_{N} q$, that is,

$$
\Delta p<2 \alpha_{N} q,
$$

due to momentum selectivity [13]. Since $\alpha_{N} \ll 1$, Eq. (18) even lowers the maximally allowed energy spread.

Further experimental challenges due to the interaction geometry and intensity fluctuations of the pump laser as well as the required properties of the electron beam taking into account three-dimensional effects are discussed in more detail in Ref. [12].

\section{Space charge vs spontaneous emission}

So far, we have considered the dynamics of a Quantum FEL governed by a unitary time evolution neglecting processes which destroy the strong correlation between momentum jumps of the electrons and the emission or absorption of photons. Decoherence mechanisms of this kind can be, for example, spontaneous emission into all modes of the radiation field [48], or space-charge effects $[49,50]$ due to the Coulomb interaction between the electrons. As these processes may eventually prevent the operation of a Quantum FEL, strong limits were imposed in Ref. [12] on the parameter regime for the electron beam and the wiggler field. We now show with the help of our results of Sec. III that these constraints can be weakened.

The discussion in Ref. [12] relied on estimating the typical length scales of the decoherence processes by classical theories. Hence, we consider the rates $k_{\mathrm{p}}$ and $R_{\mathrm{sp}}$, that is, the plasma wave number $[26,50]$ of the electron beam and the inverse decay length $[48,51,52]$, respectively, which both are listed in Table I.

To ensure a coherent time evolution over the total length $L$ of the wiggler, both processes have to occur on longer length scales than the interaction. Hence, in accordance with Ref. [12] we require the inequalities $1 /\left(2 R_{\mathrm{sp}}\right)>L$ and $1 / k_{\mathrm{p}}>$ $L$, that is, $R_{\mathrm{sp}} L<1 / 2$ and $k_{\mathrm{p}} L<1$. In addition, we demand in the quantum regime that multiphoton processes are suppressed, that is, $\alpha_{N} \ll 1$.

However, the parameters $k_{\mathrm{p}} L, R_{\mathrm{sp}} L$, and $\alpha_{N}$ are not independent of each other due to their mutual dependence on $\gamma_{0}, n_{\mathrm{e}}, \lambda_{\mathrm{W}}$, and $a_{0}$. Indeed, we can relate [12] the wiggler length $L$

$$
\frac{L}{L_{g}}=\left[12 \frac{\alpha_{N}}{\alpha_{\mathrm{f}}}\left(R_{\mathrm{sp}} L\right)\left(k_{\mathrm{p}} L\right)^{2}\right]^{1 / 3}
$$

to these three parameters and the gain length. Here we have used the definitions from Table I and introduced [53] the finestructure constant $\alpha_{\mathrm{f}} \equiv 2 \pi r_{\mathrm{e}} / \lambda_{\mathrm{C}}$.

We emphasize that the values of the parameters on the right-hand side of Eq. (19) can be chosen independently from each other. However, once this choice is made, the interaction length on the left-hand side is fixed.

In order to get an estimate for $L / L_{g}$ we set $k_{\mathrm{p}} L$ and $R_{\mathrm{sp}} L$ to their upper bounds, that is, $k_{\mathrm{p}} L=1$ and $R_{\mathrm{sp}} L=0.5$, respectively. For the example of $\alpha_{N}=0.25$ we obtain from Eq. (19) the value

$$
\frac{L}{L_{g}} \cong 5.9 \quad \text { (estimated limit) }
$$

for the maximally allowed interaction length.

However, from Eq. (11) we derive the saturation length

$$
\left.\frac{L_{\max }}{L_{g}} \cong 23.5 \quad \text { (startup from vacuum }\right)
$$

for startup from vacuum with $n_{0}=0$ and for $N=10^{9}$ electrons based on our single-mode theory. This choice for $N$ is a typical number [26,29] for electron bunches in an FEL.

Since $L \ll L_{\max }$ the coherent time evolution breaks down long before saturation is reached, and the maximally possible intensity is extremely decreased.

In contrast, for a seeded FEL with $n_{0}=0.1 \mathrm{~N}$ we deduce from Eq. (11) the saturation length

$$
\frac{L_{\max }}{L_{g}} \cong 5.1 \quad(\text { seeded FEL }),
$$

which is of the same order of magnitude as the allowed interaction length from Eq. (19) and thus the maximum intensity can be reached.

As a consequence, we believe in accordance with Ref. [12] that the focus of the research on Quantum FELs should shift from SASE to seeded FELs. We emphasize, however, that this discussion is based on arguments borrowed from classical theories and that a rigorous quantum theory covering the full interplay between multiphoton processes, spontaneous emission, and space charge is necessary to prove our statements.

Moreover, the transition to seeded FELs shifts the experimental challenges towards providing a narrow-band seed source with sufficient intensity in the x-ray regime. The lack of high-quality mirrors in this part of the spectrum impedes the construction of laser sources with sufficient power to obtain a seed with $n_{0} \sim 0.1 N$. However, one could design a hybrid scheme as proposed in Ref. [12], where a classical FEL serves as the seed for the Quantum FEL. Either the broadband output of a classical SASE FEL can be filtered and is then amplified in the Quantum FEL or a classical, (self-)seeded $\mathrm{X}$-ray FEL with powers up to the GW range [54] can be used for seeding. In the latter case, the number $n_{0}$ of photons in a single mode can attain values up to $10^{11}-10^{12}$, at least in the soft $\mathrm{x}$-ray regime according to Ref. [55]. This order of 
magnitude indicates that our example of $n_{0} \sim 10^{8}$ can be in reach of experimental capabilities.

\section{CONCLUSIONS}

In the present paper we have studied the mean intensity as well as the intensity fluctuations in a high-gain Quantum FEL. The reduction to two momentum levels limits the maximum intensity to a single emitted photon per electron. We have found that the necessary wiggler length for this maximum is significantly decreased if we consider a seeded FEL instead of SASE. Hence, the experimental realization of the former seems more feasible, especially with regard to the problematic requirements pointed out in Ref. [12]. Our results have also shown why the short wavelength of an optical undulator is necessary to fulfill the most important constraints for the operation of a high-gain Quantum FEL.

Moreover, we have observed that the time-evolved intensity fluctuations are mainly determined by the initial field state-ranging from super- to sub-Poissonian statistics in the case of a Fock or a coherent state with a high photon number, to a very broad photon distribution for vacuum.

To refine our model to more realistic scenarios we have to take space charge and spontaneous emission into all modes into account. In particular, we have to combine our many-electron theory [13] with a multimode model [52] in order to make more precise statements for the startup from vacuum. Additional to the copropagating plane wave with fixed frequency and polarization - that together with the operators $\hat{a}_{\mathrm{L}}$ and $\hat{a}_{\mathrm{L}}^{\dagger}$ define the "laser mode"-we would have to include all directions, frequencies, and polarizations; each connected to a an annihilation and creation operator. We note that each mode corresponds to a different momentum transfer on the electrons. Moreover, relativistic effects such as slippage [27] of the radiation pulse over the electron bunch have to be included, which also represent a multimode effect since a radiation pulse represents a superposition of plane waves. However, these topics go beyond the scope of our paper and are subject to further studies.

\section{ACKNOWLEDGMENTS}

We especially thank A. Debus and K. Steiniger for the fruitful collaboration with emphasis on experimental aspects of FEL physics. Moreover, we thank P. Anisimov, W. Becker, R. Endrich, A. Gover, S. Laibacher, Y. Pan, P. Preiss, and S. Varró for many exciting discussions. W.P.S. is grateful to Texas A\&M University for support from a Faculty Fellowship at the Hagler Institute of Advanced Study at Texas A\&M University, and Texas A\&M AgriLife Research for the support of his work. The research of IQ ${ }^{\mathrm{ST}}$ is financially supported by the Ministry of Science, Research and Art Baden-Württemberg.

\section{APPENDIX A: ANALYTICAL APPROXIMATION}

In this Appendix we summarize the most important steps to obtain an analytical approximation for the mean photon number in terms of Jacobi elliptic functions. Our procedure for Heisenberg operators is closely related to the one of
Ref. [32] in Schwinger representation [56] while it also leads to analogous results as other models [22,33].

\section{Dynamics of number operator}

Our analytical approach is based on the decoupling of the Heisenberg equations of motion for the photon-number operator $\hat{n}$ with the help of three constants of motion, in analogy to Ref. [32].

The time evolution of any operator $\hat{\mathcal{O}}=\hat{\mathcal{O}}(\tau)$ in the Heisenberg picture is dictated by the Heisenberg equation of motion

$$
\frac{d}{d \tau} \hat{\mathcal{O}}=i\left[\hat{H}_{\mathrm{eff}}, \hat{\mathcal{O}}\right]
$$

with the Hamiltonian

$$
\hat{H}_{\text {eff }} \equiv \varepsilon\left(\hat{a}_{\mathrm{L}} \hat{J}_{+}+\hat{a}_{\mathrm{L}}^{\dagger} \hat{J}_{-}\right)-\delta \hat{n},
$$

from Eq. (3), which is independent of the time variable $\tau$.

With the help of Eqs. (A1) and (A2), together with the commutation relations $\left[\hat{J}_{+}, \hat{J}_{-}\right]=2 \hat{J}_{z},\left[\hat{J}_{z}, \hat{J}_{ \pm}\right]= \pm \hat{J}_{ \pm}$, and $\left[\hat{a}_{\mathrm{L}}, \hat{a}_{\mathrm{L}}^{\dagger}\right]=1$ we verify that the total angular momentum

$$
\hat{A} \equiv \hat{\boldsymbol{J}}^{2}=\frac{1}{2}\left(\hat{J}_{+} \hat{J}_{-}+\hat{J}_{-} \hat{J}_{+}\right)+\hat{J}_{z}^{2},
$$

the total number of excitations

$$
\hat{B} \equiv \hat{n}+\hat{J}_{z}
$$

and the Hamiltonian

$$
\hat{C} \equiv \hat{H}_{\text {eff }}
$$

are constants of motion. We note that the three constants $\hat{A}$, $\hat{B}$, and $\hat{C}$, as well as the photon-number operator $\hat{n} \equiv \hat{a}_{\mathrm{L}}^{\dagger} \hat{a}_{\mathrm{L}}$ mutually commute with each other.

The second derivative of $\hat{n}$ with respect to time reads

$$
\frac{d^{2} \hat{n}}{d \tau^{2}}=i\left[\hat{H}_{\mathrm{eff}}, \frac{d \hat{n}}{d \tau}\right]=-\left[\hat{H}_{\mathrm{eff}},\left[\hat{H}_{\mathrm{eff}}, \hat{n}\right]\right]
$$

and after inserting $\hat{H}_{\text {eff }}$ from Eq. (A2) and calculating the nested commutator we arrive at

$$
\frac{d^{2} \hat{n}}{d \tau^{2}}=\varepsilon^{2}\left[(2 \hat{n}+1) \hat{J}_{z}+\hat{J}_{+} \hat{J}_{-}+\hat{J}_{+} \hat{J}_{-}-\frac{\delta}{\varepsilon}\left(\hat{a}_{\mathrm{L}} \hat{J}_{+}+\hat{a}_{\mathrm{L}}^{\dagger} \hat{J}_{-}\right)\right] .
$$

When we express the right-hand side purely in terms of the operators $\hat{A}, \hat{B}, \hat{C}$, and $\hat{n}$ we finally obtain the second-order differential equation [32]

$\frac{d^{2} \hat{n}}{d \tau^{2}}=-2 \varepsilon^{2}\left\{3 \hat{n}^{2}-2\left[2 \hat{B}-\left(\frac{\delta}{2 \varepsilon}\right)^{2}-\frac{1}{2}\right] \hat{n}-\left(\hat{A}+\hat{B}-\hat{B}^{2}-\frac{\delta \hat{C}}{2 \varepsilon^{2}}\right)\right\}$

for $\hat{n}$.

The dynamics of $\hat{n}$ is indeed decoupled from the electron operators. Unfortunately, we cannot solve Eq. (A8) by integration since it is a nonlinear equation of operators instead of numbers. Hence, $\hat{n}$ and $d \hat{n} / d \tau$ do not necessarily commute. 


\section{Approximating operators as $c$-numbers}

In order to find an estimate for the mean photon number $n \equiv\langle\hat{n}\rangle$ we approximate the constant operators in Eq. (A8) by their expectation values at $\tau=0$, that is, $A=r(r+1)$, $B=n_{0}+m$, and $C=-\delta n_{0}$ with $r=m=N / 2$. Here we have assumed that the field starts with the photon number $n=n_{0}$, and initially all electrons are in the excited state close to $p=q / 2$. Strictly speaking, this approximation is only valid as long as products of operators result in products of expectation values [32] when we form the total expectation value of Eq. (A8).

We then multiply the resulting $c$-number equation by $d n / d \tau$ and integrate over time $\tau$. This procedure yields the equation of motion

$$
\left(\frac{d n}{d \tau}\right)^{2}=4 \varepsilon^{2}\left(n_{+}-n\right)\left(n-n_{0}\right)\left(n+n_{-}\right),
$$

where

$$
\begin{aligned}
n_{ \pm} \equiv & \pm \frac{N}{2}\left(1-\frac{\delta^{2}}{4 \alpha_{N}^{2}}\right) \pm \frac{1}{2}\left(n_{0}-\frac{1}{2}\right)+\frac{1}{2}\left[N^{2}\left(1-\frac{\delta^{2}}{4 \alpha_{N}^{2}}\right)^{2}\right. \\
& \left.+\left(n_{0}+\frac{1}{2}\right)^{2}+2 N n_{0}\left(1+\frac{\delta^{2}}{4 \alpha_{N}^{2}}\right)+3 N\left(1+\frac{\delta^{2}}{12 \alpha_{N}^{2}}\right)\right]^{1 / 2}
\end{aligned}
$$

denotes the roots of the right-hand side of Eq. (A9).

By setting $d n / d \tau=0$ in Eq. (A9) we observe that the maximum photon number is given by $n_{+}$. The other two roots of Eq. (A9), that is, $n=n_{0}$ and $n=-n_{-}$, correspond to the minimum and initial value of $n$, and to an unphysical negative photon number, respectively.

We proceed by integrating Eq. (A9) and arrive at the expression

$$
2 \alpha_{N} \tau=\int_{n_{0} / N}^{n / N} \frac{d y}{\sqrt{\left(n_{+} / N-y\right)\left(y-n_{0} / N\right)\left(y+n_{-} / N\right)}},
$$

which describes an elliptic integral.

\section{Solution of elliptic integral}

Next we invert the elliptic integral from Eq. (A11) in order to express the mean photon number $n$ as a function of the dimensionless time $\tau$. Our result is then presented in terms of Jacobi elliptic functions [37].

These special functions are defined as inverse functions of the elliptic integral of first kind

$$
u(\varphi, \mathfrak{K}) \equiv \int_{0}^{\varphi} \frac{d y}{\sqrt{1-\mathfrak{K}^{2} \sin ^{2} y}},
$$

which is characterized by the amplitude $\varphi$ and the modulus $\mathfrak{K}$ with $0<\mathfrak{K}<1$.

There exists a set of elliptic functions, for example, sn $\equiv$ $\sin \varphi$ and $\mathrm{cn} \equiv \cos \varphi$, which are called sine amplitude and cosine amplitude, respectively. They vary between -1 and 1 and are $4 K$-periodic, with $K(\mathfrak{K}) \equiv u(\pi / 2, \mathfrak{K})$ denoting a complete elliptic integral of first kind [37].
We note that $K$ shows the asymptotic behavior [37]

$$
K \cong \ln \left(\frac{4}{\sqrt{1-\mathfrak{K}^{2}}}\right)
$$

for $\mathfrak{K}$ approaching unity, that is, $\mathfrak{K} \rightarrow 1$.

We now return to the solution of the integral in Eq. (A11) and observe for $N \gg n_{0}$ and $\delta / \alpha_{N}<2$ the relative ordering $n_{+} \geqslant n \geqslant n_{0}>-n_{-}$for the roots of the denominator. Following Ref. [37] we proceed by performing the substitution

$$
\operatorname{sn}^{2}(u, \mathfrak{K})=\frac{1}{\mathfrak{K}^{2}} \frac{y-n_{0} / N}{y+n_{-} / N},
$$

where

$$
\mathfrak{K} \equiv \sqrt{\frac{n_{+}-n_{0}}{n_{+}+n_{-}}}
$$

denotes the modulus corresponding to the sn function.

With the help of the substitution in Eqs. (A14) and (A15) we perform the integration in Eq. (A11). Solving the resulting expression for $n$ leads us finally to

$$
n=n_{0}+\left(n_{+}-n_{0}\right) \operatorname{cn}^{2}\left(\frac{L}{2 L_{g}} \sqrt{\frac{n_{+}+n_{-}}{N}}-K, \mathfrak{K}\right)
$$

after using several fundamental identities [37] for Jacobi elliptic functions. Moreover, we have expressed our result in terms of the length $L \equiv c t$ and the gain length $L_{g} \equiv c /(2 g \sqrt{N})$ by recalling the relation $\alpha_{N} \tau=L /\left(2 L_{g}\right)$ from Ref. [13].

\section{APPENDIX B: NUMERICAL SOLUTION}

In contrast to our approach in Appendix A which is based on time-dependent operators, we now employ for our numerical solution time-dependent state vectors following Ref. [36]. We first address the case of an initial Fock state and then turn to an arbitrary initial state to obtain moments of the photon distribution of a Quantum FEL.

\section{Three-term recurrence relation}

The suitable basis for the electrons in this description is given by the states $|r, m\rangle$ which fulfill the eigenvalue equations [23]

$$
\hat{\boldsymbol{J}}^{2}|r, m\rangle=r(r+1)|r, m\rangle \quad \text { and } \quad \hat{J}_{z}|r, m\rangle=m|r, m\rangle .
$$

Hence, the positive integer $r$ corresponds to the total angular momentum and $m$ to its $z$ component.

Moreover, we recall [23] the relation

$$
\hat{J}_{ \pm}|r, m\rangle=\sqrt{(r \pm m+1)(r \mp m)}|r, m \pm 1\rangle
$$

for the action of the ladder operators $\hat{J}_{ \pm}$on such a state.

Therefore, we expand the combined state vector $|\Psi\rangle=$ $|\Psi(\tau)\rangle$ of the laser field and the electrons into the basis $|n, r, m\rangle$. Here $|n\rangle$ denotes a Fock state of the laser field with $n$ photons while the eigenstate $|r, m\rangle$ for angular momentum describes the electrons.

Since the total angular momentum $\hat{A}$ as well as the total number of excitations $\hat{B}$, Eqs. (5) and (6), are constants of 
motion, we deduce that $r=N / 2=$ const and $m+n=N / 2+$ $n_{0}=$ const, respectively.

Hence, we expand the total state vector [36]

$$
|\Psi(\tau)\rangle \equiv \sum_{\nu=n_{0}}^{n_{0}+N} c_{\nu}(\tau)\left|\nu, \frac{N}{2}, \frac{N}{2}+n_{0}-\nu\right\rangle
$$

in terms of the single quantum number $v$ with the expansion coefficients $c_{v}=c_{\nu}(\tau)$. We note that $v$ varies between $v=n_{0}$ and $v=n_{0}+N$ due to the constraint $-N / 2 \leqslant m \leqslant N / 2$.

With the help of Eq. (B2) and the Schrödinger equation governed by the Hamiltonian from Eq. (3) we derive the equation of motion

$$
i \frac{d c_{v}}{d \tau}=-\delta \nu c_{v}+\alpha_{N}\left[a(v) c_{\nu-1}+a(v+1) c_{\nu+1}\right]
$$

for the probability amplitudes $c_{v}$ where the coefficients

$$
a(v) \equiv \sqrt{v\left(v-n_{0}\right)} \sqrt{1-\frac{v-n_{0}-1}{N}}
$$

are independent of $c_{\nu}$.

The solution of this linear system of differential equations relies on the diagonalization of a tridiagonal matrix with the dimension $(N+1) \times(N+1)$, which can be straightforwardly achieved by numerical methods. For this purpose, we choose the initial state $\left|n_{0}, N / 2, N / 2\right\rangle$, that is, all electrons initially are in the excited state while the laser field starts from $n=n_{0}$, which leads to the initial condition $c_{v}(0)=\delta_{v, n_{0}}$ for Eq. (B4).

\section{Arbitrary initial state}

So far, we have only considered a Fock state as initial field state. We easily generalize our approach to an arbitrary initial state characterized by the photon statistics $p_{n}$.

In the present paper, we are mainly interested in the photon statistics

$$
p_{n}^{\mathrm{coh}}=\frac{n_{0}{ }^{n}}{n !} e^{-n_{0}}
$$

and

$$
p_{n}^{\text {therm }}=\frac{1}{n_{0}+1}\left(\frac{n_{0}}{n_{0}+1}\right)^{n}
$$

of a coherent state and a thermal state both characterized by the mean photon number $n_{0}$.

The time-evolved density operator $\hat{\varrho}=\hat{\varrho}(\tau)$ of the combined system of electrons and laser field reads

$$
\hat{\varrho}(\tau)=\sum_{k, l=0}^{\infty} \varrho_{k, l} \sum_{\mu=k}^{N+k} \sum_{\nu=l}^{N+l} c_{\mu}(\tau) c_{\nu}^{*}(\tau) \hat{\mathcal{M}}(\mu, k \mid \nu, l)
$$

with the operator

$$
\hat{\mathcal{M}}(\mu, k \mid v, l) \equiv|\mu, N / 2, N / 2+k-\mu\rangle\langle v, N / 2, N / 2+l-v| .
$$

The coefficients $\varrho_{k, l}$ denote the matrix elements of the initial density operator for the laser field in photon-number representation. The diagonal elements of this matrix define the photon statistics, that is, $p_{n} \equiv \varrho_{n, n}$.

In order to calculate the expectation value of any function $f=f(\hat{n})$ of the number operator $\hat{n}$, we trace over the electrons and the laser field, that is,

$$
\langle f(\hat{n})\rangle=\operatorname{Tr}_{\mathrm{L}}\left\{\operatorname{Tr}_{\mathrm{el}}\{\hat{\varrho}(\tau)\} f(\hat{n})\right\},
$$

and arrive at the relation

$$
\langle f(\hat{n})\rangle=\sum_{n=0}^{\infty}\left\langle n\left|\left(\sum_{m=-N / 2}^{N / 2}\langle N / 2, m|\hat{\varrho}(\tau)| N / 2, m\rangle\right) f(n)\right| n\right\rangle
$$

for this expectation value.

After inserting the expression for $\hat{\varrho}$ from Eq. (B8) into Eq. (B11), and calculating the matrix element

$$
\left\langle n, \frac{N}{2}, m|\hat{\mathcal{M}}(\mu, k \mid v, l)| n, \frac{N}{2}, m\right\rangle=\delta_{n, \nu} \delta_{m, N / 2+l-v} \delta_{\mu, \nu} \delta_{k, l},
$$

we obtain the expression

$$
\langle f(\hat{n})\rangle=\sum_{n^{\prime}=0}^{\infty} p_{n^{\prime}} \sum_{\nu=n^{\prime}}^{N+n^{\prime}}\left|c_{\nu}(\tau)\right|^{2} f(\nu),
$$

which only contains the diagonal elements $p_{n^{\prime}}$.

When we set $p_{n^{\prime}}=\delta_{n^{\prime}, n}$, which corresponds to a Fock state, Eq. (B13) reduces to the formula

$$
\langle f(\hat{n})\rangle_{n} \equiv \sum_{\nu=n}^{N+n}\left|c_{\nu}(\tau)\right|^{2} f(\nu)
$$

corresponding to the case with initial photon number $n$. This result also emerges by solving Eq. (B4), and calculating the expectation value of $f(\hat{n})$ with respect to the state in Eq. (B3).

Hence, we finally obtain the elementary expression

$$
\langle f(\hat{n})\rangle=\sum_{n^{\prime}=0}^{\infty} p_{n^{\prime}}\langle f(\hat{n})\rangle_{n^{\prime}}
$$

for the expectation value of $f=f(\hat{n})$.

This result arises due to our choice of the initial state $|N / 2, N / 2\rangle$ of the electrons and due to the fact that there is only one independent quantum number, $v$.
[1] C. M. Carmesin, P. Kling, E. Giese, R. Sauerbrey, and W. P. Schleich, Quantum and classical phase-space dynamics of a free-electron laser, Phys. Rev. Research 2, 023027 (2020).

[2] C. B. Schroeder, C. Pellegrini, and P. Chen, Quantum effects in high-gain free-electron lasers, Phys. Rev. E 64, 056502 (2001).
[3] R. Bonifacio, N. Piovella, G. R. M. Robb, and A. Schiavi, Quantum regime of free electron lasers starting from noise, Phys. Rev. Spec. Top.-Accel. Beams 9, 090701 (2006).

[4] N. Piovella, M. M. Cola, L. Volpe, A. Schiavi, and R. Bonifacio, Three-Dimensional Wigner-Function Description of 
the Quantum Free-Electron Laser, Phys. Rev. Lett. 100, 044801 (2008).

[5] R. Bonifacio, H. Fares, M. Ferrario, B. W. J. McNeil, and G. R. M. Robb, Design of sub-Angstrom compact free-electron laser source, Opt. Commun. 382, 58 (2017).

[6] A. Serbeto, L. F. Monteiro, K. H. Tsui, and J. T. Mendonça, Quantum plasma fluid model for high-gain free-electron lasers, Plasma Phys. Controlled Fusion 51, 124024 (2009).

[7] M. S. Brown, J. R. Henderson, L. T. Campbell, and B. W. J. McNeil, An extended model of the quantum free-electron laser, Opt. Express 25, 33429 (2017).

[8] P. M. Anisimov, Quantum theory for 1D X-ray free electron laser, J. Mod. Opt. 65, 1370 (2018).

[9] R. Gaiba, Quantum Aspects of the Free Electron Laser, Ph.D. thesis, Universität Hamburg, 2007.

[10] P. Kling, E. Giese, R. Endrich, P. Preiss, R. Sauerbrey, and W. P. Schleich, What defines the quantum regime of the free-electron laser?, New J. Phys. 17, 123019 (2015).

[11] M. Sargent, M. O. Scully, and W. E. Lamb, Laser Physics (Addison-Wesley, Reading, MA, 1974).

[12] A. Debus, K. Steiniger, P. Kling, C. M. Carmesin, and R. Sauerbrey, Realizing quantum free-electron lasers: a critical analysis of experimental challenges and theoretical limits, Phys. Scr. 94, 074001 (2019).

[13] P. Kling, E. Giese, C. M. Carmesin, R. Sauerbrey, and W. P. Schleich, High-gain quantum free-electron laser: Emergence and exponential gain, Phys. Rev. A 99, 053823 (2019).

[14] R. H. Dicke, Coherence in Spontaneous Radiation Processes, Phys. Rev. 93, 99 (1954).

[15] W. H. Louisell, A. Yariv, and A. E. Siegman, Quantum fluctuations and noise in parametric processes. I., Phys. Rev. 124, 1646 (1961).

[16] J. K. McIver and M. V. Fedorov, Quantum theory of stimulated processes in a free-electron laser in a strong field, Zh. Eksp. Teor. Fiz. 76, 1996 (1979) [J. Exp. Theor. Phys. 49, 1012 (1979)].

[17] R. Bonifacio, Quantum SASE FEL with laser wiggler, Nucl. Instrum. Methods A 546, 634 (2005).

[18] K. Steiniger, M. Bussmann, R. Pausch, T. Cowan, A. Irman, A. Jochmann, R. Sauerbrey, U. Schramm, and A. Debus, Optical free-electron lasers with traveling-wave Thomson-scattering, J. Phys. B: At. Mol. Opt. Phys. 47, 234011 (2014).

[19] W. Becker and J. K. McIver, Fully quantized many-particle theory of a free-electron laser, Phys. Rev. A 27, 1030 (1983).

[20] E. Fernandez and M. Orszag, Many-body effects in a quantum free-electron laser model, J. Opt. Soc. Am. B 4, 512 (1987).

[21] A. Bambini and A. Renieri, The free electron laser: A singleparticle classical model, Lett. Nuovo Cimento 21, 399 (1978); A. Bambini, A. Renieri, and S. Stenholm, Classical theory of the free-electron laser in a moving frame, Phys. Rev. A 19, 2013 (1979).

[22] R. Bonifacio and G. Preparata, Quantum-mechanical description of coherent spontaneous emission, Lett. Nuovo Cimento 1, 887 (1969); Coherent Spontaneous Emission, Phys. Rev. A 2, $336(1970)$

[23] C. Cohen-Tannoudji, B. Diu, and F. Laloë, Quantum Mechanics, Volume 1 (Wiley, Singapore, 1977).

[24] We emphasize that these operators agree only formally with angular momentum, but do not describe the angular momentum of the electrons.
[25] W. Becker, M. O. Scully, and M. S. Zubairy, Generation of Squeezed Coherent States via a Free-Electron Laser, Phys. Rev. Lett. 48, 475 (1982).

[26] P. Schmüser, M. Dohlus, and J. Rossbach, Ultraviolet and Soft $X$-Ray Free-Electron Lasers (Springer, Heidelberg, 2008).

[27] R. Bonifacio, L. De Salvo, P. Pierini, N. Piovella, and C. Pellegrini, Spectrum, Temporal Structure, and Fluctuations in a High-Gain Free-Electron Laser Starting from Noise, Phys. Rev. Lett. 73, 70 (1994).

[28] P. Emma, R. Akre, J. Arthur, R. Bionta, C. Bostedt, J. Bozek, A Brachmann, P. Bucksbaum, R. Coffee, F.-J. Decker et al., First lasing and operation of an ångstrom-wavelength free-electron laser, Nat. Photonics 4, 641 (2010).

[29] C. Pellegrini, X-ray free-electron lasers: from dreams to reality, Phys. Scr. T169, 014004 (2016).

[30] M. Tavis and F. W. Cummings, Exact solution for an $N$-molecule-radiation field Hamiltonian, Phys. Rev. 170, 379 (1968).

[31] G. Scharf, Time evolution of a quantum mechanical maser model, Ann. Phys. 83, 71 (1974).

[32] S. Kumar and C. L. Mehta, Theory of the interaction of a single-mode resonant radiation field with $N$ two-level atoms, Phys. Rev. A 21, 1573 (1980).

[33] R. Gambini, Parametric amplification with a trilinear Hamiltonian, Phys. Rev. A 15, 1157 (1977).

[34] S. Carusotto, Dynamics of processes with a trilinear boson Hamiltonian, Phys. Rev. A 40, 1848 (1989).

[35] S. Haroche, Rydberg atoms and radiation in a resonant cavity, in New Trends in Atomic Physics, proceedings of the Les Houches Summer School 1982, Session XXXVIII, edited by G. Grynberg and R. Stora (North-Holland, Amsterdam, 1984), pp. 193309.

[36] D. F. Walls and R. Barakat, Quantum-mechanical amplification and frequency conversion with a trilinear Hamiltonian, Phys. Rev. A 1, 446 (1970).

[37] P. F. Byrd and M. D. Friedman, Handbook of Elliptic Integrals for Engineers and Scientists (Springer, Berlin, 1971).

[38] Based on the formalism of second quantization of G. Preparata, Quantum field theory of the free-electron laser, Phys. Rev. A 38, 233 (1988), analogous results for the mean photon number were shown in Ref. [9], at least for the startup from vacuum. In addition, we study in our paper a seeded Quantum FEL and consider higher moments of the photon statistics as well as a nonzero detuning $\delta$ from resonance.

[39] W. P. Schleich, Quantum Optics in Phase Space (Wiley-VCH, Weinheim, 2001).

[40] P. Meystre and M. Sargent, Elements of Quantum Optics (Springer, Berlin, 2007).

[41] In the classical regime the ratio of saturation and gain length is typically independent of the electron number, at least for SASE with $L_{\max }^{(\mathrm{cl})} \cong 4 \pi \sqrt{3} L_{g}^{\text {(cl) }}$ [26]. However, the gain and saturation mechanisms in the quantum regime differ from the classical ones. While for a Quantum FEL population inversion of the excited and ground state is crucial, the dynamics of its classical counterpart is based [26] on the microbunching of the electron beam.

[42] P. Kling, Theory of the Free-Electron Laser: From Classical to Quantum, Ph.D. thesis, Universität Ulm, 2018.

[43] The universal scaling is motivated by a classical theory rather than a quantum mechanical one. In contrast, our approach 
adopts a different perspective by introducing the quantum parameter $\alpha_{N}$ as the ratio of the two important frequency scales of the FEL interaction. Moreover, $\alpha_{N}$ occurs in a natural way as the expansion parameter of the asymptotic expansion in Ref. [13]. However, from an experimental point of view it is often advantageous to employ the scaling of Ref. [3].

[44] We note that for the classical case with $L_{g}^{\mathrm{cl}} \propto \gamma_{0} \lambda_{\mathrm{W}}^{1 / 3} /\left(n_{e} a_{0}^{2}\right)^{1 / 3}$ from Table I we do not face the problem of an increasing gain length since the scaling with $\gamma_{0}$ and $\lambda_{\mathrm{W}}$ is more favorable and, moreover, the condition in Eq. (15) does not have to be satisfied.

[45] R. R. Schlicher, M. O. Scully, and H. Walther, Vorschlag für einen kompakten Freie-Elektronen Laser mit einem elektromagnetischen Undulator für den Infrarot- und weichen Röntgenbereich, Technical Report, Max-Planck Institut für Quantenoptik, 1987; J. Gea-Banacloche, G. T. Moore, R. R. Schlicher, M. O. Scully, and H. Walther, Soft X-ray freeelectron laser with a laser undulator, IEEE J. Quantum Electron. 23, 1558 (1987).

[46] P. Sprangle, B. Hafizi, and J. R. Peñano, Laser-pumped coherent $\mathrm{X}$-ray free-electron laser, Phys. Rev. Spec. Top.-Accel. Beams 12, 050702 (2009).

[47] N. Piovella and R. Bonifacio, Inhomogeneous effects in the quantum free electron laser, Nucl. Instrum. Methods A 560, 240 (2006).

[48] G. R. M. Robb and R. Bonifacio, Quantum effects in spontaneous emission by a relativistic, undulating electron beam,
Europhys. Lett. 94, 34002 (2011); Coherent and spontaneous emission in the quantum free electron laser, Phys. Plasmas 19, 073101 (2012).

[49] W. H. Louisell, J. F. Lam, and D. A. Copeland, Effect of space charge on free-electron-laser gain, Phys. Rev. A 18, 655 (1978).

[50] P. Sprangle and R. A. Smith, Theory of free-electron lasers, Phys. Rev. A 21, 293 (1980).

[51] J. D. Jackson, Classical Electrodynamics (Wiley, New York, 1999).

[52] R. Endrich, Spontaneous Emission in the Quantum Regime of the Free-Electron Laser, Ph.D. thesis, Universität Ulm, 2015.

[53] For this calculation it is helpful to use the relation $g \sqrt{N}=$ $a_{0} \pi c \sqrt{2 r_{\mathrm{e}} n_{\mathrm{e}} /\left(\lambda_{\mathrm{C}} k\right)}$ for the coupling constant in the BambiniRenieri frame, which can be obtained from the definitions in Ref. [10].

[54] J. Amann, W. Berg, V. Blank, F.-J. Decker, Y. Ding, P. Emma, Y. Feng, J. Frisch, D. Fritz, J. Hastings et al., Demonstration of self-seeding in a hard-X-ray free-electron laser, Nat. Photonics 6, 693 (2012).

[55] O. Yu. Gorobtsov, G. Mercurio, F. Capotondi, P. Skopintsev, S. Lazarev, I. A. Zaluzhnyy, M. B. Danailov, M. Dell'Angela, M. Manfredda, E. Pedersoli et al., Seeded X-ray free-electron laser generating radiation with laser statistical properties, Nat. Commun. 9, 4498 (2018).

[56] J. Schwinger, On angular momentum, Technical Report, United States Atomic Energy Commission, 1952. 